\title{
Pericyte NF-kB activation enhances endothelial cell proliferation and proangiogenic cytokine secretion
}

Katherine E. LaBarbera ${ }^{1}$, Robert D. Hyldahl ${ }^{2}$, Sarah Witkowski ${ }^{1}$

${ }^{1}$ Department of Kinesiology, University of Massachusetts Amherst, ${ }^{2}$ Department of Exercise Sciences, Brigham Young University.

Correspondence: Cardiovascular and Molecular Physiology Laboratory, Department of Kinesiology, Totman Bldg., 30 Eastman Ln., University of Massachusetts, Amherst, MA 01003. Email: klabarbe@kin.umass.edu

Pericytes are skeletal muscle resident, multipotent stem cells that are localized to capillaries. They respond to damage through activation of nuclear-factor kappa-B (NF-kB), a transcription factor that regulates many cellular processes including inflammation. Research has shown that pericyte NF-KB activation positively affects myoblast proliferation. It is unknown how pericyte NF-KB affects signaling and proliferation of endothelial cells, an important component of muscle tissue microcirculation. PURPOSE: To determine the effects of altered pericyte NF-kB activity on endothelial cell proliferation and identify inflammatory factors involved in this cell-cell signaling. METHODS: Human primary pericytes were transfected with vectors designed to increase or decrease NF-KB activity (or empty vector control). Transfected pericytes were cocultured with human microvascular endothelial cells (HMVECs) using transwell inserts. HMVEC proliferation was assessed via cell counting at 24 and $48 \mathrm{hr}$. Secreted cytokines in cell culture supernatants were screened using a Luminex multiplex assay. RESULTS: HMVEC proliferation was greater in the increased pericyte NF-KB activity condition compared to the decreased NF$\mathrm{KB}$ condition at 24 and $48 \mathrm{hr}(1.3 \mathrm{fold}, \mathrm{p}=0.002)$. At $24 \mathrm{hr}$, cytokine secretion was greater in the increased NF-KB condition compared to control and decreased NF-KB conditions for 14 cytokines, including interleukin-8 (IL-8; 6.4 fold, $p<0.01$ ), monocyte chemoattractant protein-1 (MCP-1; 4.4 fold, $p<0.01$ ), and regulated on activation, normal T cell expressed and secreted (RANTES; 10.6 fold, $p<0.01$ ). CONCLUSION: NF-KB activation in pericytes caused increased HMVEC proliferation, which may have been mediated by proinflammatory and proangiogenic cytokines known to be under the transcriptional regulation of NF-KB.

Supported by: ACSM Foundation Doctoral Student Research Grant 